


\title{
A Model for Assessment and Referral of Clients with Bowel Symptoms in Community Pharmacies
}

\author{
Deepa Sriram, Alexandra McManus, Lynne M Emmerton, Richard W Parsons, Moyez \\ Jiwa
}

Doi: $10.1185 / 03007995.2015 .1135113$

\begin{abstract}
Background: To expedite diagnosis of serious bowel disease, efforts are required to signpost patients with high-risk symptoms to appropriate care. Community pharmacies are a recognised source of health advice regarding bowel symptoms. This study aimed to examine the effectiveness of a validated self-administered questionnaire, Jodi Lee Test (JLT), for detection, triage and referral of bowel symptoms suggestive of carcinoma, in pharmacies.
\end{abstract}

Method: 'Usual Practice' was monitored for 12 weeks in 21 pharmacies in Western Australia, documenting outcomes for 84 clients presenting with bowel symptoms. Outcome measures were: acceptance of verbal advice from the pharmacist; general practitioner consultation; and diagnosis. Trial of the JLT involved staff training in the research protocol and monitoring of outcomes for 80 recruited clients over 20 weeks. Utility of the JLT was assessed by post-trial survey of pharmacy staff.

Results: Significantly more referrals were made by staff using the JLT than during Usual Practice: 30 (38\%) vs 17 (20\%). Clients' acceptance of referrals was also higher for the intervention group ( $40 \%$ vs $6 \%)$. Two-thirds of pharmacy staff agreed that the JLT could be incorporated into pharmacy practice, and $70 \%$ indicated they would use the JLT in the future.

Conclusion: A pre-post design was considered more appropriate than a randomised control trial due to an inability to match pharmacies. Limitations of this study were: lack of control over adherence to the study protocol by pharmacy staff, $\backslash ;$ no direct measure of client feedback on the JLT; and loss to follow-up. The JLT was effective in prompting decision-making by pharmacy staff and inter-professional care between pharmacies and general practice, in triage of clients at risk of bowel cancer.

(C) 2015 Taylor \& Francis. This provisional PDF corresponds to the article as it appeared upon acceptance. Fully formatted PDF and full text (HTML) versions will be made available soon.



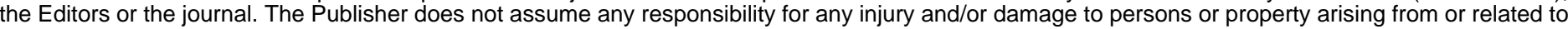



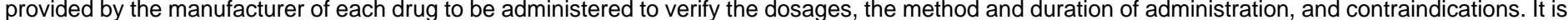

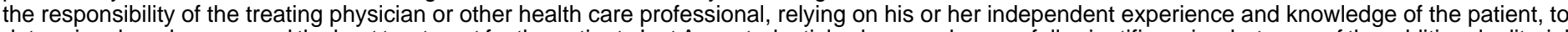

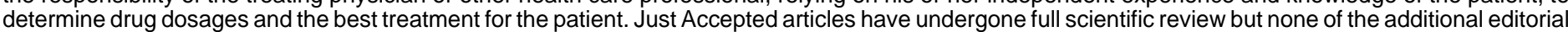

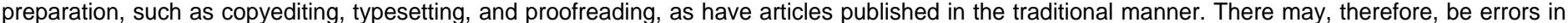

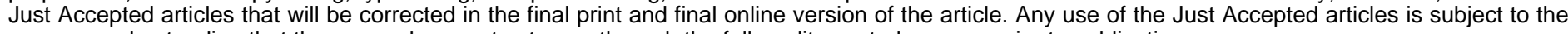
express understanding that the papers have not yet gone through the full quality control process prior to publication. 
ORIGINAL ARTICLE

A Model for Assessment and Referral of Clients with Bowel Symptoms in Community Pharmacies

Deepa Sriram, ${ }^{1}$ Alexandra McManus, ${ }^{2}$ Lynne M Emmerton, ${ }^{1 *}$ Richard W Parsons, ${ }^{1}$ Moyez Jiwa $^{3}$

1. School of Pharmacy, Faculty of Health Sciences, Curtin University, Perth, Australia

2. Centre of Excellence for Science Seafood \& Health, Faculty of Health Sciences, Curtin University, Perth, Western Australia

3. Department of Medical Education, Faculty of Health Sciences, Curtin University, Perth, Australia

Address for correspondence: Deepa Sriram, Curtin University - School of Pharmacy, Bentley Campus GPO Box U1987, Perth, Western Australia 6845, Australia. deepsri97@yahoo.com

[Running title: Assessment and Referral of Bowel Symptoms in Pharmacies]

Key words: Bowel symptoms, questionnaire, community pharmacy, referral, advice 


\section{ABSTRACT}

Background: To expedite diagnosis of serious bowel disease, efforts are required to signpost patients with high-risk symptoms to appropriate care. Community pharmacies are a recognised source of health advice regarding bowel symptoms. This study aimed to examine the effectiveness of a validated self-administered questionnaire, Jodi Lee Test (JLT), for detection, triage and referral of bowel symptoms suggestive of carcinoma, in pharmacies.

Method: 'Usual Practice' was monitored for 12 weeks in 21 pharmacies in Western Australia, documenting outcomes for 84 clients presenting with bowel symptoms. Outcome measures were: acceptance of verbal advice from the pharmacist; general practitioner consultation; and diagnosis. Trial of the JLT involved staff training in the research protocol and monitoring of outcomes for 80 recruited clients over 20 weeks. Utility of the JLT was assessed by post-trial survey of pharmacy staff.

Results: Significantly more referrals were made by staff using the JLT than during Usual Practice: $30(38 \%)$ vs $17(20 \%)$. Clients' acceptance of referrals was also higher for the intervention group (40\% vs 6\%). Two-thirds of pharmacy staff agreed that the JLT could be incorporated into pharmacy practice, and $70 \%$ indicated they would use the JLT in the future. Conclusion: A pre-post design was considered more appropriate than a randomised control trial due to an inability to match pharmacies. Limitations of this study were: lack of control over adherence to the study protocol by pharmacy staff, $\backslash$; no direct measure of client feedback on the JLT; and loss to follow-up. The JLT was effective in prompting decisionmaking by pharmacy staff and inter-professional care between pharmacies and general practice, in triage of clients at risk of bowel cancer. 


\section{A Model for Assessment and Referral of Clients with Bowel Symptoms in Community Pharmacies}

\section{INTRODUCTION}

Help seeking is considered to be the recognition of a health concern followed by a range of actions, one of which may be health service utilisation ${ }^{1}$. Interviews with people experiencing symptoms of bowel disease have shown that a significant proportion try to manage their own symptoms $^{2}$, rather than consult a doctor, even when symptoms are persistent and subsequently found to be due to a life-limiting condition ${ }^{3,4}$.

A number of bowel (colorectal) diseases share clinical presentations, and certain symptom profiles that are associated with serious underlying conditions such as cancer, inflammatory bowel disease or degenerative bowel conditions ${ }^{5}$. To improve outcomes, people need to recognise the significance of their symptoms, and general practitioners need to accurately diagnose and manage bowel diseases ${ }^{6,7}$.

Faecal occult blood test screening (FOBT), available via the National Bowel Cancer Screening Program in Australia, is limited to people turning 50, 55, 60, 65 and 74 years (the average age of a patient with colorectal cancer is 68 years $)^{8}$. Biennial screening for those aged 50-74 began in 2015 and is due to be completed in $2020^{9}$. Detection of blood in bowel motions (from the FOBT) prompts recommendations for further investigations such as colonoscopy ${ }^{9}$. At more than 30 times the price of FOBT, colonoscopy is too expensive for a population-based screening tool ${ }^{9}$. Although FOBT is a valid test for bowel cancer, participation in the program is reportedly poor ${ }^{10}$, with barriers such as: "inconvenience of the testing process; aversion to manipulating faeces; cost; views about personal invulnerability; and cultural beliefs and attitudes"11.

Community pharmacies are a recognised and common source of health advice for most Australians $^{12}$. A survey of patients attending general practice in Australia indicated that other than general practitioners, pharmacists were identified as the most likely health professional 
who might advise about bowel symptoms ${ }^{13}$. On average, three or more clients per week seek symptomatic treatment for bowel symptoms ${ }^{13}$ in each of Australia's 5450 pharmacies ${ }^{14}$. Interactions between pharmacists and their symptomatic clients therefore offer an ideal opportunity to explore how pharmacy staff can complement larger-scale screening programs by identifying emerging serious illness. However, a survey of pharmacists in Australia ${ }^{15}$ demonstrated a lack of awareness of high-risk bowel symptoms. This finding recognises the requirement for better education to understand when to refer for further medical investigation.

Research supports the use of self-administered questionnaires to help primary health care professionals, in the course of routine conversations with clients, identify cases that warrant further investigation for colorectal pathology ${ }^{16-18}$. The Jodi Lee Test (JLT) is a simple, short, client-completed questionnaire developed to aid consultation between pharmacy staff and clients with bowel symptoms ${ }^{19}$. The data provided can be reviewed by any pharmacy staff who interact with clients. For example, pharmacy assistants can use the data to determine when the client should be referred to the pharmacist, and pharmacists to determine when referral to a general practitioner is required ${ }^{19}$. The key items in the JLT indicating the need for referral for general practitioner assessment are the client's symptom(s), symptom duration and history of gastrointestinal disease ${ }^{19}$. The JLT demonstrates high sensitivity (100\%) and modest specificity $(65 \%)$ for identification and triage of symptoms of bowel disease when compared to a validated tool, the Patient Consultation Questionnaire (PCQ) ${ }^{19}$. By comparison, the PCQ assists general practitioners in prioritising referrals for colorectal conditions, and has high sensitivity for serious colorectal pathologies ${ }^{5,20}$.

The purpose of this study was to examine the feasibility and effectiveness of use of the JLT as a guide to pharmacy staff to identify clients with bowel symptoms warranting general practitioner assessment ('referral'). 


\section{MATERIALS AND METHODS}

Ethical approval was granted by the [name deleted for blind review] Human Research Ethics Committee (HR19_2013). This study used a prospective pre-post design in community pharmacies in Western Australia, and was conducted from May 2013 to March 2014. Prior to commencement of the intervention, data were collected concerning the usual practice (UP) of pharmacy staff dealing with clients seeking assistance for bowel symptoms. Following the UP phase, the JLT was introduced to guide the pharmacy staff in their interaction with the client (the intervention). These phases are described below. The value of the JLT was assessed between the two phases of the study by comparing the referrals to, and subsequent contact by the client with, the clients' general practitioner for those considered to have signs of potentially serious disease.

\section{Sample size}

The personalised data collection and client follow-up warranted a manageable, localised sample for this feasibility trial, compared to large-scale screening programs. The previous development and validation study reported that $55 \%$ of clients who were screened using the JLT were indicated as requiring general practitioner referral ${ }^{19}$. For the purposes of this study, it was conservatively assumed the referral rate would reduce to approximately $35 \%$ after the pharmacist reviewed the completed questionnaire, communicated with the client, and applied his/her clinical judgement, in line with the protocol for use of the JLT. In the previous study, approximately $10 \%$ of eligible clients were referred to their general practitioner when the pharmacist was not using a decision support aid ${ }^{13}$. In order to detect a difference of this magnitude in the proportion of clients referred for further investigation 'using' versus 'not using' the decision-support aid (35\% vs. $10 \%$ ) with power $=90 \%$ and $\alpha=0.05,65$ participants would be required in each arm of the trial. Allowing for an estimated $20 \%$ loss to follow-up, this number was adjusted to $n=82$ in each arm. Conservatively assuming that each pharmacy 
would recruit at least one participant each week, i.e. a one-in-three recruitment rate ${ }^{15}$, it was estimated that 20 pharmacies would be required to complete the study within the proposed timeframe.

A convenience sample of 21 pharmacies in Western Australia was recruited to take part in the study: 17 in the Perth metropolitan area and four from regional towns. The locations of the pharmacies were selected to represent a range of socioeconomic areas. Data collection commenced with the UP phase; however, these data were not collected from two pharmacies that joined the study prior to the commencement of the intervention. Written consent was gained from all staff members of the pharmacies, including pharmacy assistants, pharmacists, pharmacists-in-training (pre-registered pharmacists) and locums, prior to commencement of the study. Prior to commencement of each phase, author DS conducted a training session on the research protocol for the staff of each pharmacy. Instructions were given on recruitment of clients and study documentation. A written instruction sheet was left with each pharmacy for further reference, and staff were invited to report any queries or feedback. A feature of the JLT is that the pharmacist applies his/her interpretation of the questionnaire responses; as such, there was no training in clinical management of individual clients

Baseline

Staff members of the 21 intervention pharmacies completed a baseline survey comprising, a number of demographic questions and an open-ended question concerning their role in management of clients with bowel symptoms. Data were coded using key words elicited from the responses, and the roles of pharmacy assistants and pharmacists were determined collectively across the pharmacies.

Usual-Practice Phase

Pharmacy staff recruited consecutive clients seeking advice for bowel symptoms or seeking medicines normally used to treat diarrhoea, constipation or haemorrhoids. Participating clients were to be aged at least 18 years and able to give written informed consent to take part 
in the study, which included contact by the researcher for follow-up after their pharmacy visit.

The pharmacy staff continued their usual service in managing clients' bowel symptoms. Consultations with consenting clients were documented by the pharmacists, recording the clients' reported symptom(s), medication purchased, referrals for further investigation, and reasons for referrals. The pharmacies aimed to recruit and follow-up a total of 82 participants for this phase over a period of 12 weeks.

Follow-up of the recruited clients took place four weeks following their pharmacy visit. The researcher contacted clients by telephone to determine if their symptom(s) persisted, whether referrals were acted upon, and if so, investigations undertaken by the general practitioner. Verbal consent for a second follow-up at a negotiated time, was obtained from those with pending investigations to determine their ultimate diagnosis. Participants who were not contactable for follow-up after three attempts were deemed lost to follow-up.

Intervention (JLT) Phase

Recruitment started four weeks after the completion of the UP Phase. The same eligibility criteria, client recruitment and follow-up processes were used for the Intervention Phase as for the UP Phase of the study. The Intervention pharmacies were those that completed the UP Phase

In this phase, the pharmacy staff, following client consent, deployed the JLT to guide decision making in their consultation. The JLT, a paper-based questionnaire comprising eight questions, was self-completed by clients in a private or semi-private area in the pharmacy (if available), with the assistance of the staff member if required. On reviewing the completed JLT, the attending pharmacy assistant decided whether or not to refer the client to the pharmacist; likewise, the pharmacist applied his/her clinical judgement regarding referral to the client's general practitioner. For cases warranting general practitioner investigation, the 
pharmacist completed details on a standard referral letter, issued to the client with the completed JLT and verbal recommendations. Verbal recommendations included sale of medicines, instructions for use of these medicines, and relevant warnings. A carbon copy of the completed JLT was posted to the researcher in a reply-paid envelope following recruitment of each participant. The researcher contacted pharmacies twice per week by telephone and email to monitor and discuss their progress. The recruitment and follow-up period for this phase was extended to 20 weeks to account for increased pharmacy workload during December 2013 and January 2014.

Similarly to the UP Phase, clients who were referred for consultation with a general practitioner were contacted by the researcher four weeks after their pharmacy visit to determine if they had visited their general practitioner, if any further investigation had taken place, and if a diagnosis had been made.

\section{Evaluation by Staff}

Within two weeks of the completion of the intervention phase, staff of the participating pharmacies were asked to complete a post-intervention feedback questionnaire to assess the utility of the JLT. The questions included the usability of JLT, reasons why the JLT had or had not been used during the consultation, intentions concerning use of the JLT in future, if they would recommend it to their colleagues, and which (if any) other symptoms would benefit from a questionnaire such as the JLT.

Analysis

The effectiveness of the JLT intervention was determined by:

1. The proportion of clients who were referred to their general practitioner following use of the JLT compared to UP 
2. Comparison of general practitioner attendance rates for clients referred to the general practitioner following use of the JLT compared to UP

3. Diagnoses of colorectal pathologies in clients following the use of the JLT versus UP

4. Feedback from pharmacy staff on the utility of the JLT.

Demographic details of the study participants and baseline practice were summarised descriptively. Differences in referral rates and general practitioner consultations were assessed using the chi-square and Fisher's exact test. The SPSS $^{\circledR}$ version 22 statistical software was used for all analyses. A p-value $<0.05$ was interpreted as indicating a statistically significant association. 


\section{RESULTS}

Baseline

One hundred and ninety-one pharmacy staff, comprising 122 pharmacy assistants, 62 pharmacists and seven pre-registered pharmacists, completed the baseline survey. The mean age for pharmacy assistants was 28 years (range: 15-62 years), and 32 years (range: 22-56 years) for pharmacists and pre-registered pharmacists. Pharmacists and pre-registered pharmacists had around 10 years' work experience in pharmacy, while the pharmacy assistants recorded approximately six years. Twenty-one percent of the pharmacy assistants were tertiary educated, and $44 \%$ had completed year 12 or equivalent. Self-reported data from the participating pharmacies revealed pharmacy assistants typically gathered information about clients' symptoms, history and lifestyle. The pharmacists offered advice on managing symptoms and lifestyle, and provided further information about the symptoms, medication and referral to a general practitioner.

Assessment of the JLT Intervention

Eighty-four clients were recruited from 19 pharmacies and followed up over 12 weeks in the UP Phase (Table 1); these comprised 60 (71\%) females and 24 (29\%) males. Twenty-one were lost to follow-up, and seven were excluded on the basis of age.

Only 19 of the 21 selected pharmacies recruited clients for JLT phase. Eighty clients were recruited and followed up over 20 weeks in the Intervention Phase, comprising 54 (68\%) females and 26 (33\%) males. Fourteen were lost to follow-up.

Fifty of the 80 clients $(63 \%)$ were initially identified on self-completion of the JLT as meriting referral to a general practitioner. However, during the ensuing consultation, only 30 (38\%) were confirmed by pharmacists as warranting referral. Common reasons why the pharmacists did not refer cases indicated by the JLT as warranting further consideration were that the client's general practitioner was aware of the symptoms for which he/she was seeking 
advice, or that the presenting symptom was an obvious side effect of a prescription medication the client was taking.

The intervention was associated with a significantly higher referral rate during the Intervention Phase compared to the UP Phase: $38 \%$ vs $20 \%$ (Table 1). The acceptance of the recommendation to consult a general practitioner (i.e. attendance rate for general practitioner consultation) was also higher during the Intervention Phase: $40 \%$ vs $6 \%$. Three clients from each of the UP and the Intervention Phases who were referred to consult a general practitioner were lost to follow-up. The p-value (Fisher's Exact test) for comparison of the proportions of clients who were recommended to consult a general practitioner (1/14 and $12 / 27$, excluding those lost to follow-up), was 0.031 . More diagnoses were made for clients who consulted a general practitioner following the pharmacist's referral using the JLT, while there was no definitive diagnosis for the one client from the UP group who consulted a general practitioner. No diagnosis of bowel cancer was reported during the study, although several clients reported that their general practitioner had commenced monitoring.

Pharmacy Evaluation

Forty-seven pharmacy staff completed the feedback questionnaire. In this evaluation, each of the 19 participating pharmacies was represented by at least one full-time pharmacist and one pharmacy assistant. The respondents comprised 19 pharmacists and 27 pharmacy assistants. Twenty-one (45\%) reported using the JLT when consulting clients with bowel symptoms. Thirty (64\%) of the pharmacy staff agreed that the JLT could be incorporated in the pharmacy, and $33(70 \%)$ indicated they would use the JLT in future when managing clients with bowel symptoms.

The effectiveness of JLT is illustrated by the following quotations from pharmacy staff: "Effective way to establishing client needs and current bowel symptoms" (pharmacy assistant). 
“Confirming 'red flags' for early detection of bowel signs and symptoms that warranted referral for medical advice" (pharmacist).

"Establishing consistent practice in the pharmacy" (pharmacist).

"Providing timely, effective professional advice and information to clients, including advice about consulting a general practitioner" (pharmacist).

"Helpful in reinforcing the case of referral when the client was initially hesitant to see the general practitioner" (pharmacist).

Additional feedback related to the JLT being simple and quick to use, easy to understand by the client, non-invasive, easier for clients who feel embarrassed to discuss their bowel symptoms, and a good checklist approach for quick response in a busy pharmacy. In critiquing the tool, some stated the study protocol booklet format was time consuming and a deterrent to the recruitment process. Reasons given by staff for not using the JLT were largely logistical, including workflow, lack of private consultation space in their pharmacy, and few clients perceived as eligible. Other conditions for which the pharmacy staff would accept a JLT-like questionnaire were urinary tract infections, asthma, vaginal candidiasis, cough, chronic pain, kidney problems, headaches, and upper gastro-intestinal symptoms. 


\section{DISCUSSION}

This prospective study supports the use of a brief self-administered questionnaire, the JLT, as a clinical decision tool for pharmacy staff to identify symptoms that might require medical investigation. It also serves as a written referral to the general practitioner. Capitalising on the personalised nature and convenience of pharmacist-client consultations, this intervention has the potential to complement larger-scale bowel cancer screening programs. Staff in the pharmacy were made aware of people who were presenting with symptoms that may require medical assessment. The client and the pharmacist were afforded the opportunity to discuss these in detail, and in some cases, referral was considered unnecessary. A reasonable assumption was made by the pharmacists that if a client had recently consulted a general practitioner, the general practitioner was almost certainly aware of the symptom(s) and was managing the client appropriately. Other clinical situations may have also overridden a pharmacist's decision to refer a client.

In cases where referral was warranted, the participants were advised of the need for a medical consultation and given a referral letter to take to the general practitioner. Use of the JLT resulted in $38 \%$ of clients being referred to their general practitioner, compared to $20 \%$ during UP. This result is in line with studies reporting that health questionnaires completed by patients frequently captured more positive symptoms than elicited during consultation ${ }^{21,22}$. Our empirical evidence also indicates a greater proportion of the clients accepted referral. This finding is consistent with other studies that reported increased general practitioner consultation after being encouraged by a pharmacist ${ }^{13,23}$.

The concept of applying a decision-support tool in pharmacy practice was novel to our participating pharmacy staff, and although it may not be applicable to all practice settings or situations, the JLT shows promise in guiding management of bowel symptoms. In particular, the documentation of completed JLT and written referral, produced for cases warranting referral appears to hold value for clients, evidenced by their uptake of recommendations to 
consult their general practitioner. Alternatively, for situations able to be managed in the pharmacy, the JLT highlights to the pharmacy staff member the presenting symptoms and their significance.

As established in the baseline phase, the first point of contact for the client was commonly the pharmacy assistant. As such, prompting pharmacy assistants' use of a simple, structured assessment tool can benefit the pharmacy workflow in triaging clients to be referred to the pharmacist and supporting the provision of non-prescription medicines; these are recognised roles for pharmacy assistants in Australia ${ }^{24}$.

Most of the pharmacy staff found the JLT to be a simple and effective assessment guide for management of bowel symptoms, and reported that clients managed to complete the questions unassisted. As such, its deployment did not burden pharmacy staff. The majority of burden related to client recruitment and consent for research purposes (follow-up).

Although a randomised control trial (RCT) is the ideal design to test pharmacy-led interventions; it was not feasible in this case for several reasons. Firstly, one of the objectives of the study was to identify if a change in referral practices of pharmacists could be achieved by introducing to them a structured questionnaire approach (the JLT) for these clients. Once the JLT is used for a particular client in a pharmacy, it would not be practical to revert to UP for a subsequent client. A cluster design where pharmacies applied only UP or the JLT would have avoided the issue of randomising clients, but differences between practices in terms of staffing and demographic profile may have confounded the analysis. For these reasons, a prepost design was considered the most appropriate.

The study protocol was not consistently applied in some pharmacies, highlighting the challenges of research in a naturalistic setting. Although the researcher closely monitored the study progress, adherence to the study protocol by individual staff was not able to be controlled. Ideally, this trial would have also included client feedback on the JLT to 
supplement developmental research in its design ${ }^{19}$, and validation of client outcomes using general practice data. Further research on the acceptability of the JLT directly from the client's perspective is required to determine their expectations of pharmacy services when presenting with bowel symptoms. Though a practising GP was involved in the design and validation stage of $\mathrm{JLT}^{19}$, no GPs to whom the clients were referred to, were asked to give feedback on the written referral that was given to clients whose symptoms warranted further medical consultation. Loss to follow-up is also recognised as a limitation in outcomesfocussed research.

Our findings suggest potential for wider application of the JLT as an optional practiceenhancing guide to over-the-counter consultations in the community pharmacy setting. There is potential for the documentation to be adapted to guide management of other complex symptoms potentially warranting general practitioner investigation and potentially associated with early-stage cancer. There is potential for future research on development and trial of a JLT-like questionnaire for screening of pharmacy clients presenting with symptoms indicative of conditions such as urinary tract infection, vaginal candidiasis and kidney problems. 


\section{CONCLUSIONS}

The JLT was found to be an acceptable assessment tool for the triage of bowel symptoms in a pharmacy setting. Its effectiveness was demonstrated by prompting a higher rate of referrals in those who would benefit from a general practitioner investigation, a higher rate of uptake of recommendations for referral and more clinical diagnoses compared to the usual model of consultation. As such, the JLT shows promise as an effective decision-making aid in the pharmacy to triage clients at higher risk of bowel cancer.

\section{TRANSPARENCY}

Declaration of funding:

Author DS was supported by a Jodi Lee Foundation PhD scholarship.

Declaration of financial/other relationships:

All authors have disclosed that they have no relevant financial relationships. CMRO Peer Reviewers on this manuscript have no relevant financial or other relationships to disclose.

Author contributions:

All authors were involved in the study design. DS conducted the study. RP provided statistical guidance with analysis and interpretation of data. All authors drafted and revised the manuscript.

\section{Acknowledgements:}

The authors acknowledge the participation of local pharmacists and pharmacy assistants in data collection. 


\section{REFERENCES}

1. Smith J, Braunack-Mayer A, Wittert G. What do we know about men's help-seeking and health service use? Med J Aust 2006;184(2):81-3.

2. Oberoi D, Jiwa M, McManus A, et al. Help-seeking experiences of men diagnosed with colorectal cancer: a qualitative study. Eur J Cancer Care 2015 doi: 10.1111/ecc.12271. 3. Bain NSC, Campbell NC, Ritchie LD, Cassidy J. Striking the right balance in colorectal cancer care - a qualitative study of rural and urban patients. Fam Pract 2002;19(4):369.

4. Ramos M, Arranz M, Taltavull M, et al. Factors triggering medical consultation for symptoms of colorectal cancer and perceptions surrounding diagnosis. Eur J Cancer Care 2010;19(2):192-9.

5. Ballal M, Selvachandran S, Maw A. Use of a patient consultation questionnaire and weighted numerical scoring system for the prediction of colorectal cancer and other colorectal pathology in symptomatic patients: A prospective cohort validation study of a Welsh population. Colorectal Dis 2010;12(5):407-14.

6. Ott J, Ullrich A, Miller A. The importance of early symptom recognition in the context of early detection and cancer survival. Eur J Cancer 2009;45(16):2743-8.

7. Mitchell E, Macdonald S, Campbell N, et al. Influences on pre-hospital delay in the diagnosis of colorectal cancer: a systematic review. Br J Cancer 2008;98(1):60-70.

8. McGrath DR, Leong DC, Gibberd R, et al. Surgeon and hospital volume and the management of colorectal cancer patients in Australia. ANZ J Surg 2005;75(10):901-10. 9. Australian Government Department of Health. National Bowel Cancer Screening Program. Available from

http://www.cancerscreening.gov.au/internet/screening/publishing.nsf/Content/about-theprogram-1 [Last accessed 15 May 2015]

10. Australian Institute of Health and Welfare. Australia's health 2008. Available from http://www.aihw.gov.au/publication-detail/?id=6442468102 [Last accessed 2 March 2015]

11. Australian Cancer Network Colorectal Cancer Guidelines Revision Committee.

Guidelines for the Prevention, Early Detection and Management of Colorectal Cancer.

Available from https://www.nhmrc.gov.au/guidelines-publications/cp106. [Last accessed 2 March 2015]

12. Benrimoj SI, Roberts AS. Providing Patient Care in Community Pharmacies in Australia. Ann Pharmacother 2005;39(11):1911-7. 
13. Jiwa, Sargant S, Hughes J, O'Connor M, Hodder R. Triaging consumers who present bowel symptoms to community pharmacies: a pilot study of two interventions. Aust Pharm 2009;28:516-20.

14. Pharmacy Guild of Australia. Welcome. Available from http://www.guild.org.au/. [Last accessed 21 April 2015]

15. Jiwa M, Spilsbury K, Duke J. Do pharmacists know which patients with bowel symptoms should seek further medical advice? A survey of pharmacists practicing in community pharmacy in Western Australia. Ann Pharmacother 2010;44(5):910-7.

16. Adelstein B-A, Irwig L, Macaskill P, Katelaris PH, Jones DB, Bokey L. A self administered reliable questionnaire to assess lower bowel symptoms. BMC Gastroenterol 2008;8(1):8.

17. Jiwa M, Hughes J, Sriram D, Birring S, Meng X, Cecchele G, et al.Piloting and validating an innovation to triage patients presenting with cough to community pharmacies in Western Australia. Qual Primary Care 2012;20(2):83-91.

18. Selvachandran S, Hodder R, Ballal M, Jones P, Cade D. Prediction of colorectal cancer by a patient consultation questionnaire and scoring system: a prospective study. Lancet 2002;360(9329):278-83.

19. Sriram D, McManus A, Emmerton L, Parsons R, Jiwa M. Development and validation of a clinical decision-making aid for screening bowel symptoms in community pharmacies. J Eval Clin Pract 2014;20(3):260-6.

20. Rai S, Ballal M, Thomas W, Miller A, Jameson J, Steward W. Assessment of a patient consultation questionnaire-based scoring system for stratification of outpatient risk of colorectal cancer. Br J Surg 2008;95(3):369-74.

21. Fromme EK, Eilers KM, Mori M, Hsieh Y-C, Beer TM. How accurate is clinician reporting of chemotherapy adverse effects? A comparison with patient-reported symptoms from the Quality-of-Life Questionnaire C30. J Clin Oncol 2004;22(17):3485-90.

22. Justice A, Chang C, Rabeneck L, Zackin R. Clinical importance of provider-reported HIV symptoms compared with patient-report. Med Care 2001;39(4):397-408.

23. Atchan M, Davis D, Foureur M. Applying a knowledge translation model to the uptake of the Baby Friendly Health Initiative in the Australian health care system. Women Birth 2014;27(2):79-85.

24. Pharmacy Board of Australia. Guidelines for dispensing of medicines. Available from http://www.pharmacyboard.gov.au/Codes-Guidelines.aspx. [Last accessed 21 April 2015] 
Table 1. Impact Evaluation: Usual-Practice Phase versus Intervention Phase

\begin{tabular}{|c|c|c|}
\hline Variable & Usual Practice & Intervention \\
\hline Recruited & 84 & 80 \\
\hline $\begin{array}{l}\text { Referred to } \\
\text { General } \\
\text { practitioner }\end{array}$ & $17(20 \%)$ & $30(38 \%)^{*}$ \\
\hline $\begin{array}{l}\text { Consulted General } \\
\text { practitioner }\end{array}$ & $1 / 17(6 \%)$ & $12 / 30(40 \%)^{* *}$ \\
\hline $\begin{array}{l}\text { Details of General } \\
\text { practitioner } \\
\text { Consultation }\end{array}$ & $\begin{array}{l}\# 55 \text { : No follow-up } \\
\text { for diagnosis }\end{array}$ & $\begin{array}{l}\# 05: \text { Monitored by general practitioner } \\
\# 07: \text { Ultrasound } \rightarrow \text { diverticulitis } \\
\# 13: \text { Blood tests } \rightarrow \text { monitored by general } \\
\text { practitioner } \\
\# 26: \text { No further action } \\
\text { \#41: No further action } \\
\# 51: \text { Dairy allergy } \\
\text { \#55: Monitored by general practitioner } \\
\# 58: \text { No follow-up for diagnosis } \\
\# 74: \text { Blood and stool test } \rightarrow \text { all clear } \\
\# 76: \text { Stool test } \rightarrow \text { all clear } \\
\# 80: \text { Colonoscopy } \rightarrow \text { monitored by general } \\
\text { practitioner } \\
\# 83: \text { Colonoscopy } \rightarrow \text { monitored by general } \\
\text { practitioner }\end{array}$ \\
\hline
\end{tabular}

* $\mathrm{p}=0.029$ (chi-square)

$* * \mathrm{p}=0.017$ (Fisher's Exact) 